\title{
ARTICLE
}

Received 21 Oct 2013 | Accepted 20 Feb 2014 | Published 19 Mar $2014 \quad$ DOl: 10.1038/ncomms4476

\section{Jasmonic acid regulates spikelet development in rice}

Qiang Cai ${ }^{1, \star}$, Zheng Yuan ${ }^{1, \star}$, Mingjiao Chen ${ }^{1}$, Changsong Yin ${ }^{1}$, Zhijing Luo ${ }^{1}$, Xiangxiang Zhao ${ }^{2}$, Wanqi Liang${ }^{1}$, Jianping $\mathrm{Hu}^{3} \&$ Dabing Zhang ${ }^{1}$

The spikelet is the basal unit of inflorescence in grasses, and its formation is crucial for reproductive success and cereal yield. Here, we report a previously unknown role of the plant hormone jasmonic acid (JA) in determining rice (Oryza sativa) spikelet morphogenesis. The extra glume 1 (eg1) and eg2 mutants exhibit altered spikelet morphology with changed floral organ identity and number, as well as defective floral meristem determinacy. We show that EG1 is a plastid-targeted lipase that participates in JA biosynthesis, and EG2/OsJAZ1 is a JA signalling repressor that interacts with a putative JA receptor, OsCOI1b, to trigger OsJAZ1's degradation during spikelet development. OsJAZ1 also interacts with OsMYC2, a transcription factor in the JA signalling pathway, and represses OsMYC2's role in activating OsMADS1, an E-class gene crucial to the spikelet development. This work discovers a key regulatory mechanism of grass spikelet development and suggests that the role of JA in reproduction has diversified during the flowering plant evolution.

\footnotetext{
${ }^{1}$ State Key Laboratory of Hybrid Rice, School of Life Sciences and Biotechnology, Shanghai Jiao Tong University, Shanghai 200240, China. ${ }^{2}$ Jiangsu Key Laboratory for Eco-Agricultural Biotechnology around Hongze Lake, Huaiyin Normal University, Huian, Jiangsu, 223300, China. ${ }^{3}$ Department of Energy Plant Research Laboratory, Michigan State University, East Lansing Michigan, 48824, USA. ${ }^{\star}$ These authors contributed equally to this work. Correspondence and requests for materials should be addressed to D.Z. (email: zhangdb@sjtu.edu.cn).
} 
$\mathrm{R}$ ice (Oryza sativa) is a major staple food for half of the world population and a model plant to investigate evolutionary developmental biology in grasses, monocot plants that compose one of the largest families in angiosperms ${ }^{1}$. One unique feature of grasses is that each plant contains specialized spikelets, structural units of inflorescences that show great morphological diversity even within the grass family ${ }^{2}$.

Compared with dicot plants, grasses evolved more types of specialized axillary meristems during reproduction. For instance, rice initiates branch meristem from the inflorescence meristems, produces spikelet meristem from inflorescence branches and converts spikelet meristem to floral meristem ${ }^{3}$. Each rice spikelet contains two pairs of leaf-like structures: rudimentary glumes and sterile lemmas, and a single fertile flower that consists of a lemma, a palea, two lodicules, six stamens and one pistil ${ }^{4}$. Rice spikelet morphogenesis is pivotal to the determination of inflorescence architecture and seed production.

In Arabidopsis thaliana, floral meristem arises from inflorescence meristem in response to multiple flowering signals, such as the phytohormone auxin ${ }^{5}$. Auxin-responsive transcription factor MONOPTEROS (MP) induces the expression of a floral meristem identity gene, $L E A F Y(L F Y)$, which is also regulated by other flowering regulators including FLOWERING LOCUS T (FT), SUPPRESSOR OF OVEREXPRESSION OF CONSTANS 1 (SOC1), and AGAMOUS-LIKE 24 (AGL24), resulting in the transition from inflorescence meristem to floral meristem ${ }^{5,6}$. $L F Y$ and APETALA1 (AP1) induce each other's expression to initiate flower primordium emergence and morphogenesis ${ }^{7,8}$. Unlike their counterparts in Arabidopsis ${ }^{9}$, rice AP1-like and $L F Y$-like genes are involved in inflorescence meristem specification ${ }^{10-12}$. Instead, OsMADS1/LEAFY HULL STERILE1 (LHS1), an E-class gene, functions similarly to $L F Y$ and $A P 1$ in determining floral meristem initiation and specification ${ }^{13,14}$. Loss-of-function of OsMADS1 leads to reiterative formation of glumes or extra spikelet formation within spikelets, and defective organ identity $^{13,15}$. In contrast to Arabidopsis, in which APETALA2 $(A P 2)$ genes play a role in specifying floral organ identity ${ }^{9}$, rice, maize and possibly other grasses use $A P 2 / E R F$-like genes to control spikelet meristem transition and deteminacy ${ }^{16-19}$. These findings suggest that grasses and dicots seem to use the same sets of genes in different aspects of meristem identity specification in reproduction.

Jasmonic acid (JA) and its derivatives are lipid-derived hormones that control plant defence response and developmental events, such as seed germination, root growth, tuber formation, tendril coiling, trichome initiation, reproduction and senescence $^{20-24}$. Two Arabidopsis phospholipases, DONGLE (DGL) and DEFECTIVE IN ANTHER DEHISCENCE1 (DAD1), release $\alpha$-linolenic acid (C18:3) from chloroplastic membrane lipids as the first step of JA biosynthesis ${ }^{25,26}$. Recent evidence showed that DGL and DAD1 are not essential for wound- or pathogeninduced JA biosynthesis and that other lipases may be involved in stress-induced JA production, suggesting that plants may use different lipases for jasmonate biosynthesis in various biological processes $^{2 f}$. In the well-characterized Arabidopsis JA signalling pathway, JA is perceived by the receptor CORONATINEINSENSITIVE1 (COI1), an F-box protein that activates the expression of JA-responsive genes by degrading the JAZ (jasmonate ZIM-domain) repressors ${ }^{28-35}$. Despite our understanding of JA biosynthetic and signalling pathways, relatively little is known about the precise functions of JA in plant (particularly crop) development.

In this study, we characterized, extra glume 1 (eg1) and eg2, two rice mutants with aberrant spikelet morphology, and identified the EG1 and EG2 genes through fine-mapping. EG1 is a lipase in JA biosynthesis and EG2/OsJAZ1 is a repressor of the JA signalling pathway. We further showed that EG2/OsJAZ1 interacts with the putative JA receptor, OsCOIlb, and with the transcription factor OsMYC2, to repress OsMYC2's function in activating the E-class gene OsMADS1 in spikelet development. This work reveals a unique role of JA in regulating rice spikelet development, which is to specify floral organ identity and floral meristem determinacy.

\section{Results}

eg1-3 and eg2-1D exhibit abnormal spikelet morphology. To understand the mechanism that controls rice spikelet development, we identified two spikelet morphological mutants with similar phenotypes, and named them extra glumes (eg) 1-3 and eg2-1D. Both mutants displayed defects in spikelet development, including extra glume-like structures between the sterile lemma and lemma and altered floral organ numbers and identities (Fig. 1a, Supplementary Fig. 1a-g and Supplementary Table 1). Genetic analyses suggested that eg1-3 was caused by a single recessive mutation (wt:eg1-3 $=78: 27, \chi^{2}=0.03, P>0.05, \chi^{2}$-test used), whereas eg2-1D was caused by a single dominant mutation (wt:eg2-1D=30:87, $\chi^{2}=0.03, P>0.05, \chi^{2}$ test used).

To characterize the spikelet defects in eg1-3 and eg2-1D, we performed detailed histological observations (Fig. 1b). The spikelets of eg1-3 and eg2-1D contained palea that exhibited lemma-like characteristics, such as five vascular bundles, no mrps and opened flower hull (Fig. 1b and Supplementary Fig. 1c-g). In addition, both mutants displayed transformation of lodicules into glume-lodicule mosaic structures (Fig. 1a,b), and decreased number of stamens and pistil (Supplementary Table 1). To determine the genetic relationship between EG1 and EG2, we constructed eg1-3 eg2-1D double mutant. The double mutant showed phenotypes similar to the two single mutants but to stronger degrees. It had lost floral organ identity, displaying more glume-like structures outside the lemma and palea, homeotic transformation of floral organs to glume-like structures and in some cases, extra spikelets with elongated pedicels at their centre (Fig. 1a,b and Supplementary Fig. 1h).

Further analysis using scanning electron microscopy revealed that eg1-3 and eg2-1D had early developmental defects in spikelet. In wild-type spikelet, after the differentiation of a pair of sterile lemma primordium, the spikelet meristem was converted into floret meristem and produced one lemma and one palea primordium (Fig. 1c and Supplementary Fig. 2a). However, the spikelets of eg1-3 and eg2-1D developed extra glume-like structures after the formation of sterile lemma primordium, which resulted in additional floral whorls (Fig. 1c,d and Supplementary Fig. 2b-h). Floral meristem determinacy was also markedly affected in eg1-3 and eg2-1D, which had bulged meristem and ectopic floral organ primordium and ectopic glume-like structures (Fig. 1c and Supplementary Fig. 2b-h). Moreover, compared with the singe mutant, eg1-3 eg2-1D grew more ectopic floral primordium, glume-like structures or double floral meristem in one spikelet, suggesting defects in floral meristem indeterminacy (Fig. 1c and Supplementary Fig. 2i-1). Together, these results suggested that EG1 and EG2 may be functionally associated or even act in the same pathway that determines rice floral meristem determinacy and floral organ identity.

EG1 encodes a lipase for JA biosynthesis. The EG1 gene was mapped to chromosome 1 between two In-Del markers, CQ1 and Os116 (Fig. 2a). Sequence analysis revealed that eg1-3 contains a G-to-A substitution in the coding region of an annotated gene ${ }^{36}$ (LOC_Os01g67430, http://www.gramene.org/), causing the change of Gly to Arg at the 216th amino acid (Fig. 2b). Besides 
a
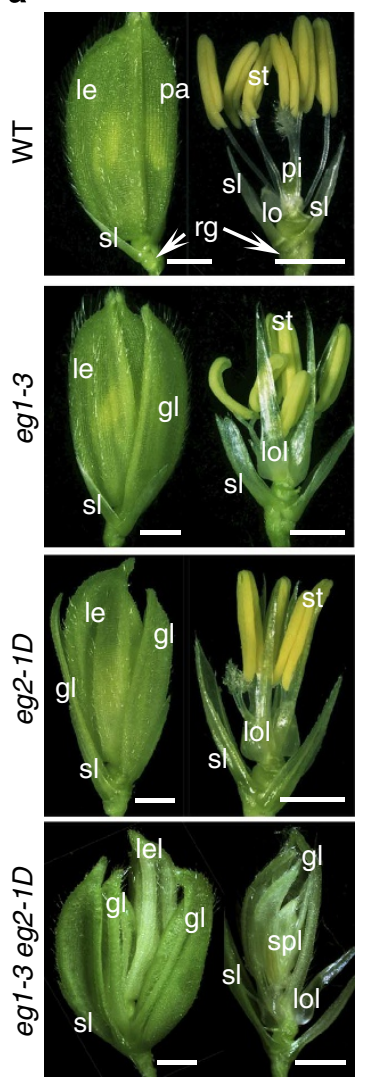

b
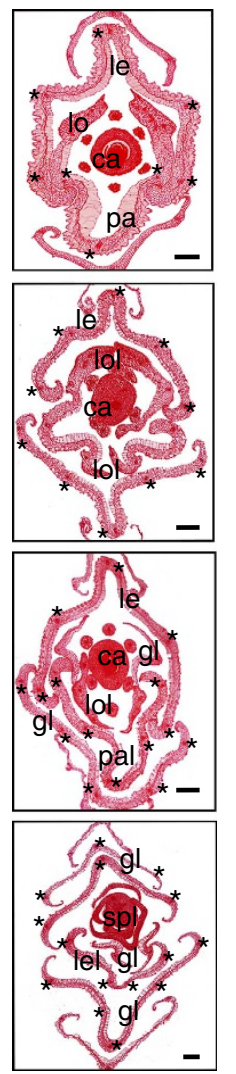

C
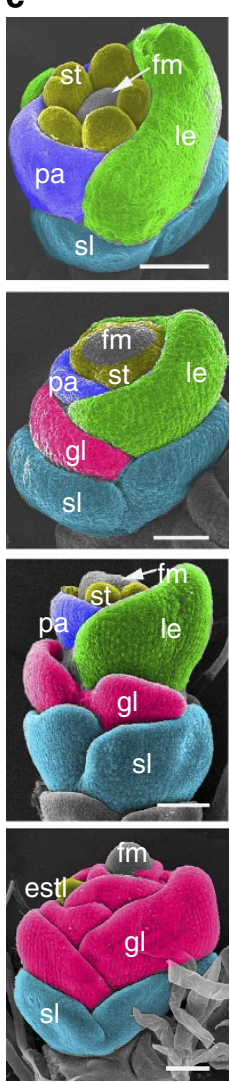

d
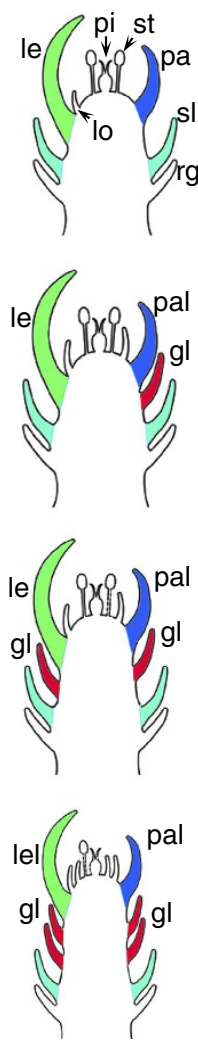

Figure 1 | Phenotypic analysis of spikelet development in mutants. (a) Rice spikelets. Scale bars, $1 \mathrm{~mm}$. (b) Transverse paraffin sections of spikelets. Stars indicate vascular bundles. Scale bars, $100 \mu \mathrm{m}$. (c) Scanning electron microscopic pictures of floral organ primordium. Organ identities are indicated by pseudocolors: sterile lemma in navy blue, lemma in green, palea in blue, stamen in light yellow and ectopic glumes in red. Scale bars, $50 \mu m$. (d) Diagrams of the spikelets, showing position and growth pattern of floral organs. estl, ectopic stamen-like; gl, glume-like; le, lemma; lel, lemma-like; lo, lodicule; lol, lodicule-like; pa, palea; pal, palea-like; pi, pistil; rg, rudimentary glume; sl, sterile lemma; spl, spikelet-like; st, stamen.

ubiquitous expression in seedlings, roots, shoot and leaves during vegetative growth, EG1 also expressed at reproductive stage, in inflorescence meristem, spikelet meristem and floral organs (Supplementary Fig. 3), further supporting the role of EG1 in spikelet development.

Genetic analysis demonstrated that eg1-3 was allelic to eg1-1 and eg1-2 (Fig. 2c,d), two spikelet development mutants identified from a previous study, which showed that the stronger allele (eg1-1) grew ectopic floral organs at each organ whorl or in extra ectopic whorls, resulting in an indeterminate floral meristem ${ }^{36}$. This previous study identified EG1's role in rice spikelet development, yet the biochemical function of EG1 was not investigated. EG1 belongs to class $I$ of the phospholipase $A_{1}$ family ${ }^{36}$, in the same clade as the homologous Arabidopsis JA biosynthetic enzymes DGL and DAD1 (refs 25,26). To test whether EG1 is an enzyme in JA production, we overexpressed EG1 cDNA in the Arabidopsis dad1 mutant, and observed rescue of the male sterile and silique development phenotypes (Fig. 3a). Conversely, expression of $E G 1_{\text {pro: }} D G L$ in eg1-3 also rescued the abnormal spikelet phenotypes (Fig. $3 \mathrm{~b}$ ). Thus, EG1 is the rice ortholog of DAD1 and DGL, functioning as a lipase in JA production during rice spikelet development.

Using the rice protoplasts and Nicotiana benthamiana leaf cells as protein expression systems, we observed localization of EG1-GFP (green fluorescent protein) in chloroplasts (Supplementary Fig. 4), where DAD1 and DGL function ${ }^{25,26}$. Measurement of endogenous JA levels showed that compared with the wild type, the total amount of free JA in eg1-3 decreased
28, 32 and $54 \%$ in seedling, inflorescence and spikelets, respectively (Fig. $3 \mathrm{c}$ ). In agreement with the previously reported function of JA in repressing root growth ${ }^{24}$, root length of eg1-3 was also increased (Supplementary Fig. 5). Exogenous application of MeJA rescued the abnormal spikelet (Fig. 3d,e) and longer root (Supplementary Fig. 5) phenotypes in the eg1-3 mutant, further confirming that the spikelet development defect in eg1-3 was caused by reduced content of JA resulted from the lack of a functional EG1 protein in JA biosynthesis.

EG2 is a repressor of JA response and interacts with OsCOI1b. The EG2 gene was mapped to chromosome 4 between CQQ2 and CQQ3, two In-Del markers that define a $2.2 \mathrm{cM}$ (centimorgan) genomic region (Fig. 4a). Sequence analysis identified a mutation in LOC_Os04g55920 (http://www.gramene.org/) in eg2-1D (Fig. 4b). We named the gene OsJAZ1 because of its high sequence similarity with the Arabidopsis JAZ proteins. The mutation in eg2-1D was a C-to-G transition, resulting in a substitution of Ala by Gly in the fifth amino acid of the Jas domain (Fig. 4b), a motif known to be critical for JAZ's interaction with the JA receptor COI1 (refs 28-31).

To confirm that the mutated OsJAZ1 is responsible for defective spikelet development in eg2-1D, a plasmid containing a 6.6-kb OsJAZ1 genomic fragment amplified from eg2-1D

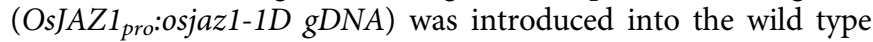
and eg1-3 (Fig. 4c-i). Transgenic lines exhibited abnormal spikelets similar to those in eg2-1D (Fig. 4c-e,i) and lines with 
a

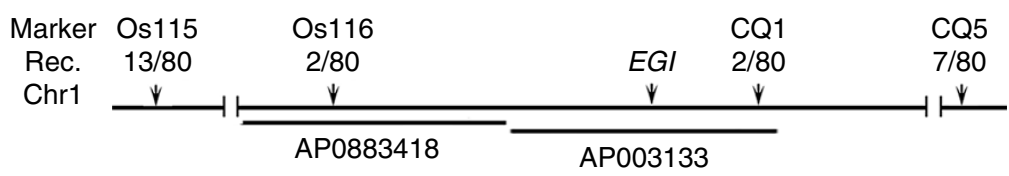

b
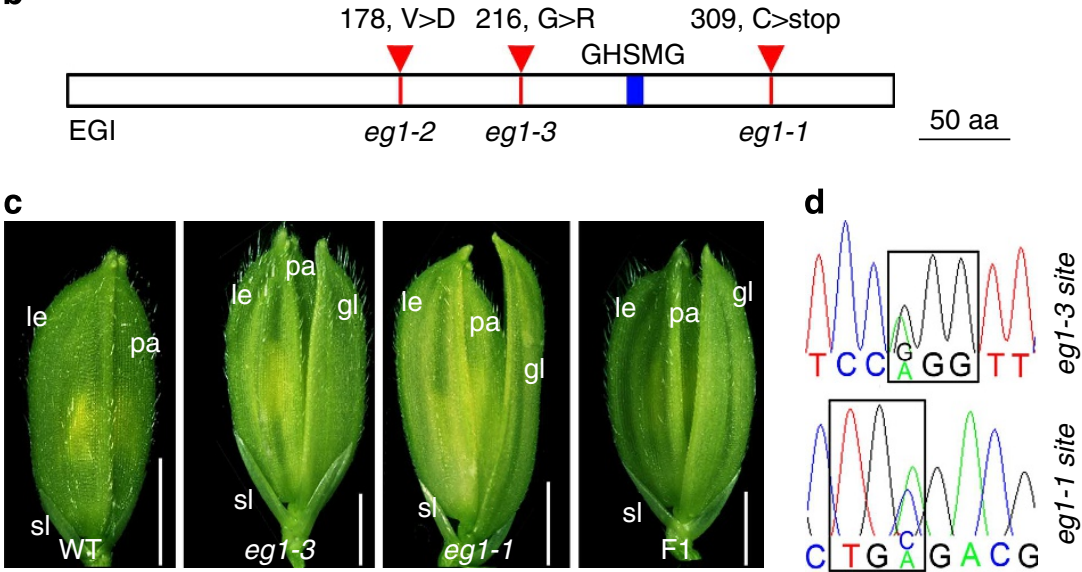

Figure 2 | Positional cloning of EG1. (a) The location of EG1 on the BAC clone AP003133 on chromosome 1. (b) A schematic representation of the EG1 protein. The mutant alleles are indicated by red lines and triangles. The conserved lipase III domain (GMSMG) is indicated by a blue bar. (c) Spikelet morphology of the wild type, eg1-3, eg1-1 and $F_{1}$ progeny from eg1-3 $x$ eg1-1. Scale bars, $1 \mathrm{~mm}$. (d) Sequence analysis of the EG1 gene in $F_{1}$ progeny, showing mutated nucleotides in the mutant alleles. gl, glume-like; le, lemma; pa, palea; sl, sterile lemma.

higher level expression of osjaz1-1D exhibited stronger phenotypes, that is, having multiple layers of glume-like structures in the spikelet (Fig. $4 \mathrm{f}, \mathrm{i}$ ). In addition, the eg1-3 OsJAZ1 pro:osjaz1-1D $g D N A$ lines showed stronger phenotypes than eg1-3, mimicking the phenotype of eg1-3 eg2-1D double mutants (Fig. $4 \mathrm{~g}-\mathrm{i}$ ). These results demonstrated that the abnormal spikelet development in eg2-1D was caused by the mutation in the Jas domain of OsJAZ1. EG2 expressed ubiquitously in the plant, from roots, stems and leaves, to spikelets and seeds (Supplementary Fig. 6), overlapping with the expression pattern of EG1, further supporting the notion that these two proteins may function in the same pathway.

To investigate whether OsJAZ1 is involved in JA signalling, we treated the eg2-1D-mutant seedlings with MeJA. Unlike the wild type, the eg2-1D mutant was insensitive to jasmonate's inhibition of root growth (Supplementary Fig. 7), a phenotype similar to those reported for Arabidopsis jai3-1 mutant that was defective in the $J A Z 3$ gene and transgenic lines overexpressing the JAZ1 gene deleted for the Jas domain ${ }^{28,32}$.

To further characterize the role of OsJAZ1 in JA response, we used yeast two-hybrid $(\mathrm{Y} 2 \mathrm{H})$ assays to test the interaction between OsJAZ1 and three rice COI1-like proteins: OsCOI1a, OsCOI1b and OsCOI1 $c^{37}$. OsJAZ1 and OsCOI1b interacted, and the interaction was dependent on JA-Ile's mimic, coronatine (COR), but not JA or MeJA (Fig. 5a). This JA-Ile-dependent interaction is similar to what was reported in Arabidopsis between JAZ proteins and COI1 (refs 28-32). No interaction was observed between OsJAZ1 and OsCOIla or OsCOI1c (Fig. 5a). Furthermore, the Ala-to-Gly mutation in the Jas domain of OsJAZ1 in eg2-1D blocked osjaz1-1D-OsCOI1b interaction (Fig. 5b), suggesting that the Jas domain is required for the interaction between OsJAZ1 and OsCOI1b.

To test how OsJAZ1 affects spikelet development, we generated transgenic plants overexpressing OsJAZ1-GUS and osjaz1-1DGUS fusion proteins, respectively. After 1 -h treatment of $100 \mu \mathrm{M}$ MeJA, GUS signals were greatly reduced in the OsJAZ1-GUS transgenic plants and the addition of $100 \mu \mathrm{M}$ MG132, a specific inhibitor of the $26 \mathrm{~S}$ proteasome, prevented JA-mediated elimination of the GUS signals (Fig. $5 c$ and Supplementary Fig. 8). By contrast, GUS signals were stable after JA treatment in the osjaz1-1D-GUS transgenic lines (Fig. 5c and Supplementary Fig. 8), suggesting that the osjaz1-1D in eg2-1D rendered the protein resistant to JA-induced proteasome-mediated degradation. In addition, overexpression of osjaz1-1D-GUS, but not OsJAZ1-GUS, resulted in defective spikelet development in the wild type, similar to the phenotype of eg1-3 and eg2-1D (Fig. 5d), suggesting that OsJAZ1 is a key component of the JA signalling pathway during rice spikelet development and its regulation is similar to that of the Arabidopsis JAZ proteins.

JA pathway affects the expression of E-class genes. To uncover downstream components in the pathway by which JA regulates spikelet development in rice, we analysed the expression of possible target genes in the eg1-3 and eg2-1D mutants. In rice, five E-class (SEPALLATA, SEP) genes (OsMADS1, OsMADS5, OsMADS7, OsMADS8 and OsMADS34) play a key role in specifying inflorescence and spikelet development ${ }^{38-40}$. The osmads 1 mutant and OsMADS1/5/7/8-RNAi lines display spikelet defect similar to eg1-3 eg2-1D double mutant and some osjaz1-1D gain-of-function lines (Figs $1 \mathrm{a}$ and $4 \mathrm{f})^{15,38}$. Thus, we analysed the expression of OsMADS1, OsMADS5, OsMADS7, OsMADS8 and OsMADS34 in the eg1-3 and eg2-1D mutants by quantitative reverse transcriptase-PCR (qRT-PCR). The expression of OsMADS1, OsMADS7 and OsMADS8 was reduced in eg1-3 and eg2-1D mutants at early stages of spikelet development (Fig. 6a), but no obvious changes were detected for OsMADS5 and OsMADS34 (Supplementary Fig. 9). In addition, in situ hybridization analysis of the eg1-3 and eg2-1D mutants revealed that the expression signal of OsMADS1 became much weaker in lemma and palea primordium and the expression of OsMADS7 and OsMADS8 was reduced in the floral meristem (Fig. 6b). Furthermore, expression analysis showed close correlation between higher expression level of osjaz1-1D and reduced expression level of OsMADS1, OsMADS7 and OsMADS8 

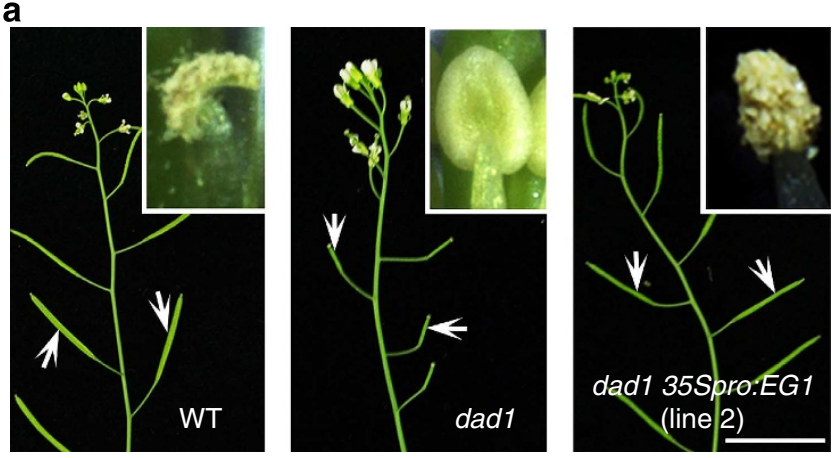

b

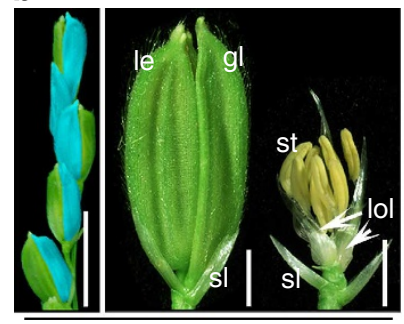

eg1-3
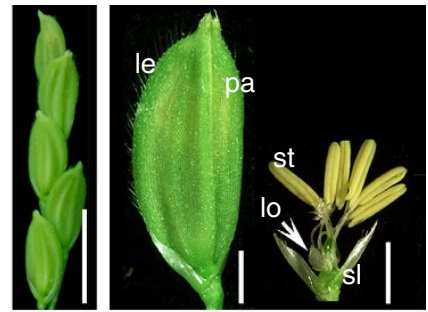

eg1-3 EG1 pro: $_{\text {DGL (line1) }}$

C

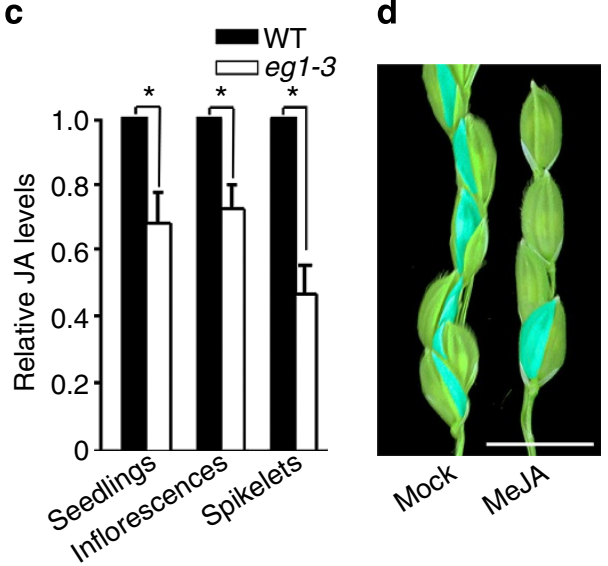

e

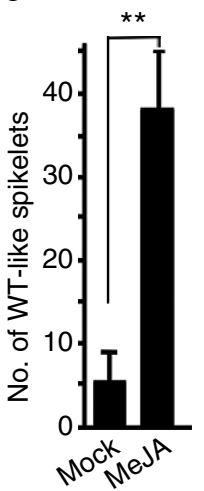

Figure 3 | EG1 is involved in JA production. (a) Overexpression of EG1 in the dad1 mutant rescued anther indehiscence (insets) and silique development (arrows) phenotypes. Scale bar, $1 \mathrm{~cm}$. (b) The defects in eg1-3 spikelets were rescued by expressing $E G 1_{\text {pro: }} D G L$. Sky blue indicate extra glumes. Scale bars, $1 \mathrm{~cm}$ for the branches, $1 \mathrm{~mm}$ for spikelets.

(c) Comparison of endogenous JA content in the wild type and eg1-3. The aerial part of 6-day-old seedlings, 2-4 mm long inflorescence and spikelets at late stage of Sp8 were used. Each data point is the average of three biological repeats, and error bars indicate s.d. Asterisks indicate significant difference between samples (Student's $t$-test $P$-values, ${ }^{\star} P<0.05$;

${ }^{\star}{ }^{*} P<0.01$ ). (d) Exogenous application of MeJA rescued the abnormal spikelet development phenotype in eg1-3. Sky blue indicates extra glumes in eg1-3 spikelets. Scale bar, $1 \mathrm{~cm}$. (e) Quantification of wild type-like spikelets in the inflorescence after MeJA treatment in (d). Each data point is the average of 10 biological repeats, and error bars indicate s.d. Asterisks indicate significant difference between samples (Student's $t$-test $P$-values, ${ }^{\star} P<0.05$; $\left.{ }^{\star \star} P<0.01\right)$. gl, glume-like; le, lemma; lo, lodicule; lol, lodicule-like; pa, palea; sl, sterile lemma; st, stamen.

in OsJAZ1 $1_{\text {pro: }}$ osjaz1-1D gDNA transgenic lines (Supplementary Fig. 10). These results suggest that JA regulates early spikelet development possibly by activating the expression of OsMADS1, OsMADS7 and OsMADS8.
To check the genetic interaction between these SEP-like proteins and JA signalling pathway, we crossed eg1-1 with the available mutant, osmads $1-z^{39}$. Similar to the phenotype in osmads1- $z$ and OsMADS1/5/7/8-RNAi lines, eg1-1 osmads1- $z$ double mutant and osmads1- $z$ mutant containing OsJAZ1 $1_{\text {pro:os- }}$ jaz1-1D gDNA showed elongated lemma and palea, and homeotic transformation of inner floral organs into glume-like structures (Fig. 6c,d). These data suggested that OsMADS1 (and possibly OsMADS7 and OsMADS8) is downstream from the action of EG1 and OsJAZ1 in the JA pathway.

OsMYC2 directly regulates OsMADS1 expression. The Arabidopsis basic helix-loop-helix transcription factor MYC2 is a crucial activator of JA response genes ${ }^{28,41,42}$. To determine whether rice MYC2 homologues are involved in JA signalling in spikelet development, we cloned the only rice homologue of Arabidopsis MYC2, OsMYC2 (ref. 43), which was highly expressed in spikelet and floral organs (Supplementary Fig. 11), and tested its interaction with OsJAZ1 using $\mathrm{Y} 2 \mathrm{H}$ assays. Both OsJAZ1 and osjaz1-1D interacted with OsMYC2, and the interaction required the Jas domain, as the deletion of this domain abolished the interaction (Fig. 7a,b). Bimolecular fluorescence complementation assays and pull-down assays also showed that OsMYC2 interacted with full-length OsJAZ1, and the mutation in the Jas domain in osjaz1-1D did not affect this interaction (Fig. 7c,d). These results demonstrate that OsMYC2 may function as a direct target of OsJAZ1 in spikelet development.

The basic region of the Arabidopsis MYC2 protein binds to the G-box motif (5'-CACGTG/CACATG- $\left.3^{\prime}\right)$ in the promoters of MYC2 target genes ${ }^{42}$. There are five G-box motifs in the OsMADS1 genomic region; G1 and G2 are in the promoter and G3, G4, and G5 are in the first intron (Fig. 8a). To determine whether OsMADS1 is a target of OsMYC2 in transcription, we produced antibody against OsMYC2 (Methods) and performed chromatin immunoprecipitation (ChIP)-PCR assays. In wild-type spikelet, G1 and G2, but not G3, G4 or G5, can be amplified from immunoprecipitation pulled down by the anti-OsMYC2 antibody (Fig. 8b). Yeast one-hybrid assays and DNA-binding assays further revealed that OsMYC2 was able to bind to the G2 region but not G1 in the OsMADS1 promoter (Fig. 8c,d). Furthermore, dual-luciferase (dual-LUC) assays showed that the expression level of luciferase driven by the OsMADS1 promoter was transiently increased by OsMYC2, but this increase was abolished when OsJAZ1 was co-expressed (Fig. 8e), indicating that OsJAZ1 interacts with OsMYC2 and suppresses the activity of OsMYC2 in triggering the transcription of OsMADS1. These results suggested that OsMYC2 directly binds to the OsMADS1 promoter, at least through G2, and thus it directly regulates the expression of OsMADS1.

\section{Discussion}

The grass spikelet displays diverse morphologies among taxa, and its structure is a key determinant of grain production, yet the developmental and genetic mechanisms underlying this great diversity remain largely unclear. In this work, we discovered a novel regulatory mechanism in rice spikelet development that is dependent on JA (Fig. 9).

Results from genetic complementation and JA measurements suggested that, like its orthologs in Arabidopsis, rice EG1 is an important enzyme in JA biosynthesis. However, EG1's expression does not seem to be induced by wounding (Supplementary Fig. 12), which differs from its Arabidopsis conterparts ${ }^{25,26}$. Based on these data, we propose that EG1 is largely if not exclusively dedicated to JA production in rice development and not in 
a

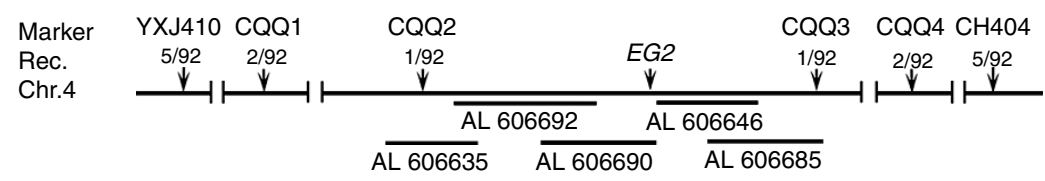

b

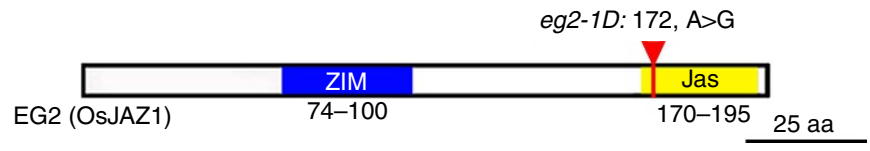

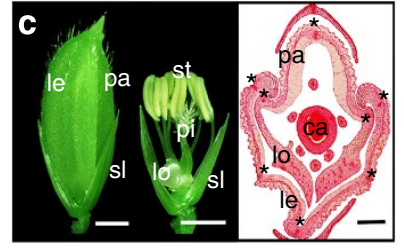

WT
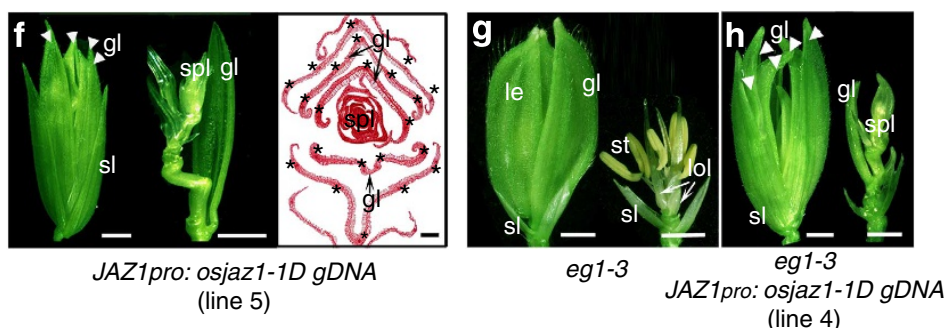

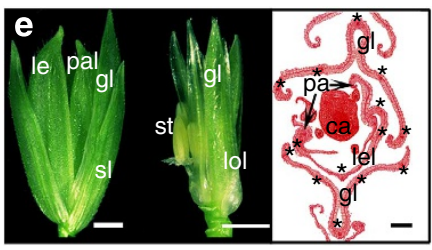

JAZ1pro: osjaz1-1D gDNA (line 3)

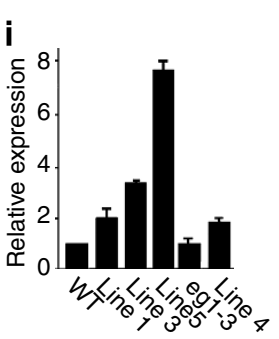

Figure 4 | Positional cloning of EG2 (OsJAZ1). (a) The location of EG2 (OsJAZ1) on chromosome 4 . (b) A schematic representation of the EG2 protein. (c-h) Spikelets of transgenic lines expressing OsJAZ1 pro:0sjaz1-1D gDNA in the wild-type (d-f) and eg1-3 (h) background. Arrowheads indicate

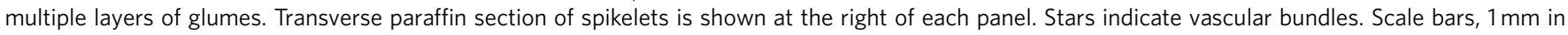
the spikelet images, $100 \mu \mathrm{m}$ in the transverse paraffin sections. (i) Quantitative analysis of the expression of OsJAZ1/osjaz1-1D in spikelets in (c-h). Inflorescences were 3-4 mm, corresponding to stage Sp6 in the wild type. The expression level of OsJAZ1 in the wild type was set as 1. Each data point represents the average of three biological repeats, and error bars indicate s.d. gl, glume-like; le, lemma; lo, lodicule; lol, lodicule-like; pa, palea; pal, palea-like; pi, pistil; spl, spikelet-like; st, stamen.

wounding response. The fact that the endogenous JA level in eg1-3 mutant decreased $\sim 50 \%$ compared with the wild type suggests that there might be unidentified genes encoding JA producing enzymes in rice spikelet development.

The role of JA in rice spikelet development is confirmed by the identification of the mutant of EG2/OsJAZ1, which encodes a protein homologous to the Arabidopsis JAZ proteins. Biochemical and genetic evidence in this study uncovered the role of JA in promoting the degradation of OsJAZ1 via the OsCO1b-mediated proteasome pathway. This mode of action for OsJAZ1 is similar to that of the Arabidopsis JAZ proteins ${ }^{28-32}$. No protein-protein interaction between OsJAZ1 and OsCOI1a or OsCOI1c was observed, possibly because that OsCOIla and OsCOIlc interact with other JAZ proteins and regulate JA's action in other biological events. Rice genome contains $15 \mathrm{JAZ}$ members and the expression of OsJAZ1 and eight other JAZ genes was detected in rice spikelet at stage Sp4 (Supplementary Fig. 13), suggesting the possible redundant role of JAZ members in rice spikelet development, similar to the redundant role of Arabidopsis JAZ proteins ${ }^{24}$.

Besides the spikelet defects, both eg1-3 and eg2-1D exhibit phenotypes during vegetative development, such as longer roots and larger leaves in seedlings (Supplementary Fig. 14), confirming the previously reported role of JA in inhibiting vegetative organ growth $^{24}$. However, eg1-3 and eg2-1D showed no obvious changes in the height and leaf size of adult plants and inflorescence branch development, possibly due to functional redundancy of other JA biosynthetic and signalling genes.
Phenotypes of eg1-3 and eg2-1D in spikelet morphology clearly differ from that of JA defective mutants reported in other species $^{21}$. For example, Arabidopsis mutants impaired in JA biosynthesis and perception, such as OPDA reductase 3 (opr3), dad1, allene oxide synthase (aos), fatty acid desaturation ( $f a d$ ) 3-2 fad7-2 fad8 and coil, exhibit male sterility caused by insufficient filament elongation, non-viable pollen and delayed anther dehiscence $^{21}$. In contrast to the male sterile phenotype of Arabidopsis coil mutant ${ }^{44}$, mutants of the tomato COI1 homologue are female sterile with defective maternal control of seed maturation ${ }^{45}$, indicating that JA signalling plays distinct roles in flower development in Arabidopsis and tomato. In maize (Zea mays), Tasselseed1 (TS1) encodes a plastidtargeted lipoxygenase with predicted 13-lipoxygenase activity and participates in JA biosynthesis ${ }^{46,47}$. Two maize oxophytodienoate reductase genes, $O P R 7$ and $O P R 8$, were shown to participate in JA production ${ }^{48}$. Both $t s 1$ and $o p r 7$ opr 8 mutants convert staminate to pistillate in tassel inflorescence, indicating that JA is required for male sex determination and suppression of female reproductive organ biogenesis in maize $e^{46-48}$. Based on these results from other plant species and our own data, we propose that the conserved JA biosynthetic and signalling pathways play an important role in distinct aspects of reproductive development in various plant species, targeting different downstream genes.

Control of the spikelet meristem transition and determinacy is a key event during grass inflorescence morphogenesis, and 
a

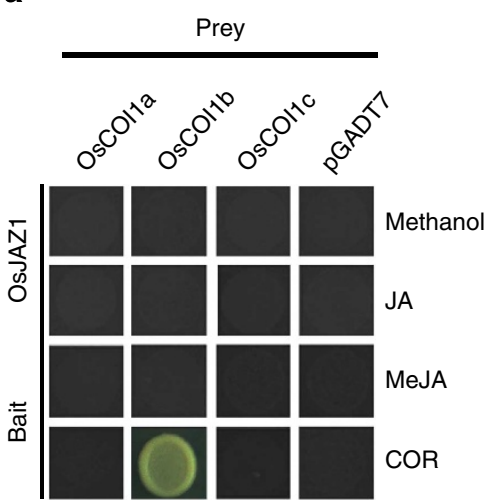

C

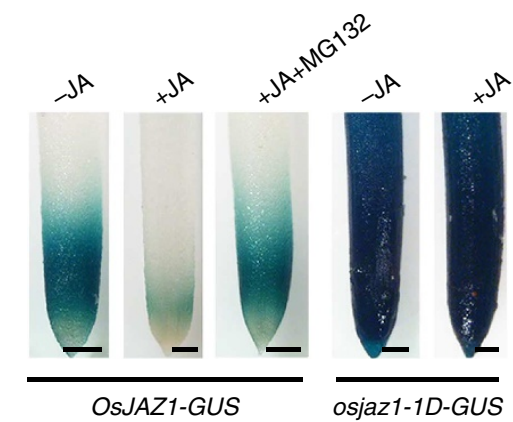

b
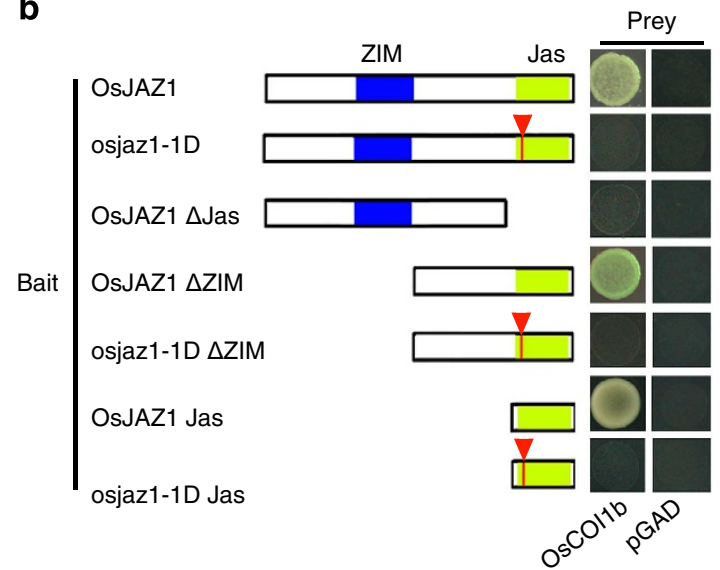

d

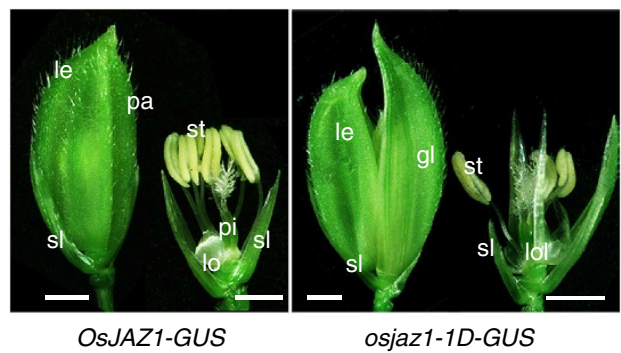

Figure 5 | OsJAZ1 is involved in JA signalling and acts as a repressor of spikelet development. (a) $Y 2 \mathrm{H}$ assays to test interaction between OsJAZ1 and three rice COI1 homologues in the presence of JA derivatives. (b) $\mathrm{Y} 2 \mathrm{H}$ analysis showing the coronatine (COR)-dependent OsJAZ1-OsCOI1b interaction. Red and red line triangle indicates the mutation in osjaz1-1D. (c) JA-dependent proteasome-mediated degradation of OsJAZ1-GUS and osjaz1-1D-GUS in roots. MG132, a proteasome inhibitor. Scale bars, $1 \mathrm{~mm}$. (d) Spikelets of transgenic lines expressing 35S pro:OsJAZ1-GUS and 35S pro:0sjaz1-1D-GUS in wild-type background. Scale bars, $1 \mathrm{~mm}$. gl, glume-like; le, lemma; lo, lodicule; lol, lodicule-like; pa, palea; pi, pistil; sl, sterile lemma; st, stamen.

successful formation of floral organs and fertile flowers determines grain production. To date, several proteins have been identified to restrict the development of axillary meristems within the spikelet meristem and subsequently specify meristem identity. These proteins include the grass ethylene response factor transcription factors, such as maize BRANCHED SILKLESS 1 (BD1) and rice FRIZZY PANICLE (FZP), AP2 transcription factors such as maize INDETERMINATE SPIKEKET 1 (IDS1), SISTER of INDETERMIATE SPIKELET 1 (SID1), rice SUPERNUMERARY BRACT (SNB), OsIDS1 and MULTI-FLORET SPIKELET 1 (MFS1), and Brachypodium distachyon MORE SPIKELETS $^{49}$. However, the roles of AP2 gene products in the specification of spikelet meristem fate in monocot are distinct from that of their homologues in Arabidopsis, which is to specify floral organ identity ${ }^{9}$. Furthermore, LONG STERILE LEMMA (G1), a member of a plant-specific gene family that contains proteins with an uncharacterized ALOG (Arabidopsis LSH1 and Oryza G1) domain, specifies rice spikelet morphology by determining sterile lemma identity ${ }^{50}$. TONGARI-BOUSHI 1 (TOB1), a YABBY protein, controls proper lateral organ development and spikelet meristem maintenance in a noncell-autonomous manner ${ }^{51}$. Whether the JA pathway interacts with these genes in regulating rice spikelet development remains to be elucidated.

In the past decade, investigations suggest that the classical ABCDE model generated from studies of dicots, including Arabidopsis, Petunia hybrida and Antirrhinum majus, is partially applicable to grasses such as rice and maize ${ }^{52}$. Grasses evolved diversified E-class (SEPALLATA $[S E P])$ genes $^{52}$. Rice has at least five SEP members: OsMADS1/LEAFY HULL STERILE1 (LHS1), OsMADS5, OsMADS7, OsMADS8 and OsMADS34 (ref. 52). The lhs1/osmads1 mutant displays changed floral meristem identity ${ }^{13}$ Transgenic plants silenced for both OsMADS7 and OsMADS8 show late flowering, floral organ homeotic transformations and floral indeterminacy ${ }^{38}$. OsMADS34/PANICLE PHYTOMER2 (PAP2) regulates the development of inflorescences and spikelets 39,40 . Emerging evidence suggests that ancient AGAMOUS-LIKE6 (AGL6)-like genes conserved in gymnosperns and angiosperms, such as rice OsMADS6/MOSAIC FLORAL ORGANS1 (MFO1 $)^{53-55}$ and maize zea agamous3 $(z a g 3)^{56}$, the close homologues of SEP-like genes, have SEP-like functions essential for flower development. In this study, we show a previously unreported JA pathway that regulates the expression of OsMADS1, OsMADS7 and OsMADS8 in rice spikelet development, linking the upstream JA pathway to the floral meristem identity genes (Fig. 9). In this pathway, OsMYC2, the only rice homologue of Arabidopsis MYC2 (ref. 43), binds to the $\mathrm{G} 2$ site in the promoter of OsMADS1. The phenotype of eg1-3 eg2-1D double mutant resembles that of OsMADS1/5/7/8-RNAi lines $^{38}$ and the eg1-1 Osmads1-z double mutant. That the eg1-3 or eg2-1D single mutant displays more moderate phenotypes compared with that of OsMADS1/5/7/8-RNAi transgenic plants may be due to the redundant role of other JA pathway genes besides EG1 and EG2, and/or the presence of other signalling pathway(s) that control the expression of OsMADS1/7/8 during rice spikelet development. Whether JA signalling regulates other genes in spikelet development remains to be elucidated in the future.

In summary, we present a role of JA in regulating rice floral meristem determinacy and floral organ identity (Fig. 9). The mechanism discovered in this study may be applicable to many other grasses with similar spikelet structure, including species in 
a

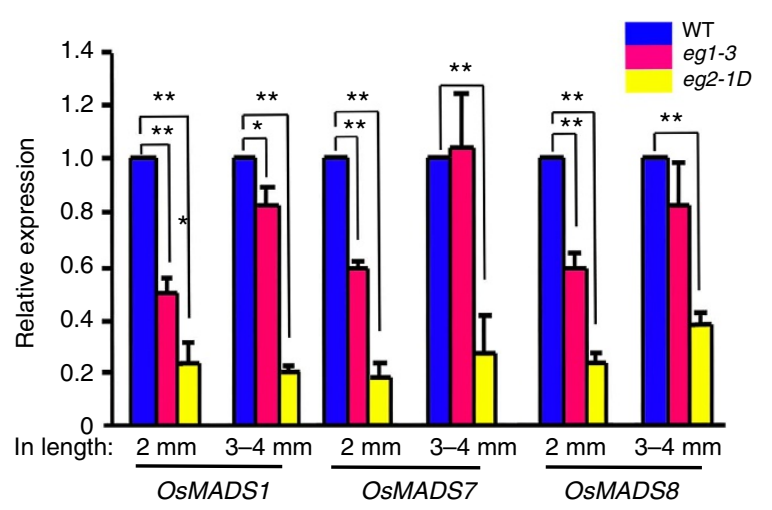

b

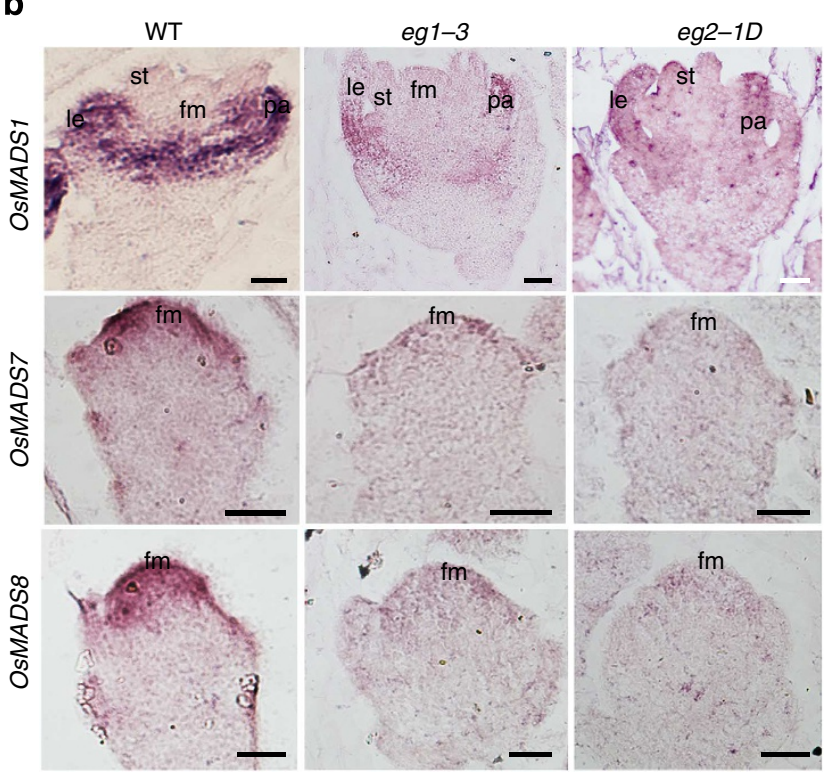

d

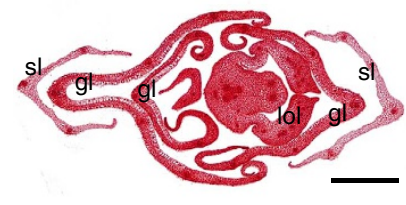

osmads $1-z$

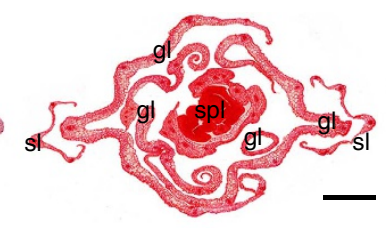

eg1-1 osmads 1-z c
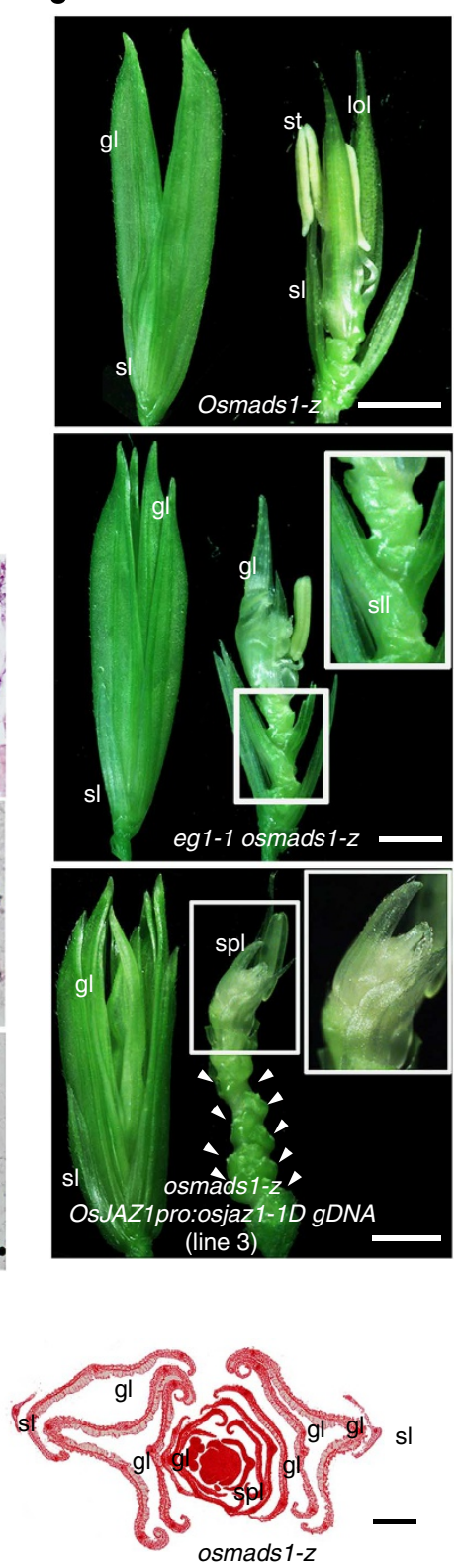

OsJAZ1pro:osjaz1-1D gDNA

(line 3)

Figure 6 | JA signalling regulates the expression of three E-class genes in spikelets. (a) Quantitative analysis of OsMADS1, OsMADS7 and OsMADS8 transcripts in $2 \mathrm{~mm}$ (stage Sp4 in wild type) and 3-4 mm (stage Sp6 in wild type) inflorescences in the wild type, eg1-3 and eg2-1D. Actin expression in each sample was used as a control. The expression level in the wild type was set as 1. Each data point represents the average of three biological repeats, and error bars indicate s.d. Asterisks indicate significant difference between samples (Student's -test $P$-values, ${ }^{\star} P<0.05$; $\left.{ }^{\star \star} P<0.01\right)$. (b) In situ analysis of the expression of three OsMADS genes in wild-type, eg1-3 and eg2-1D spikelets. Scale bars, $25 \mu \mathrm{m}$. (c) Spikelet phenotypes of osmads1-z, eg1-1 osmads1-z and osmads1-z OsJAZ1 pro:0sjaz1-1D gDNA. Insets are magnified images of the boxed regions to show spikelet-like structure formed in the centre of the spikelet. Arrowheads indicate the position of the extra whorls of glumes (which had been removed before images were taken) in the spikelet. Scale bars, $1 \mathrm{~mm}$. (d) Transverse paraffin section of wild-type and mutants spikelets in (c). Scale bars, $100 \mu \mathrm{m}$. fm, floral meristem; gl, glume-like; in, inflorescences; le, lemma; lol, lodicule-like; pa, palea; sl, sterile lemma; sll, sterile lemma-like; spl, spikelet-like; st, stamen.

Oryza, Sorghum and Panicum, and provides basis to efforts aimed at improving crop yield.

\section{Methods}

Plant growth conditions and analyses of spikelet development. The strains eg1-3, eg2-1D and osmads1- $z$ were in background of 9522 (wild type, O. sativa L. ssp. japonica), eg1-1 was in Zhefu 802 background (wild type, O. sativa L. ssp. indica). eg1-3 and eg2-1D mutants were isolated by screening an ethyl-methane sulphonate-induced mutant library. osmads $1-z$ was from our previous work ${ }^{39}$. eg1-1 was kindly provided by Prof. Y.B. Xue ${ }^{36}$. The dad1 mutant (Salk_025432, in Col-0 background) was obtained from the Arabidopsis Biological Resource Center (http://www.arabidopsis.org/). All rice plants were grown in the isolated paddy field in Shanghai Jiao Tong University in the summer.

The development stages of rice spikelet were defined by Ikeda et al. ${ }^{57}$. The histological, transverse section and scanning electron microscopic analyses were performed as described by Li. et al. ${ }^{55}$.

Generation of transgenic lines. To generate transgenic $E G 1_{\text {pro: }}$ GUS ( $\beta$-glucuronidase) lines, the $2-\mathrm{kb} E G 1$ promoter region was cloned into PCAMBIA1301 
a

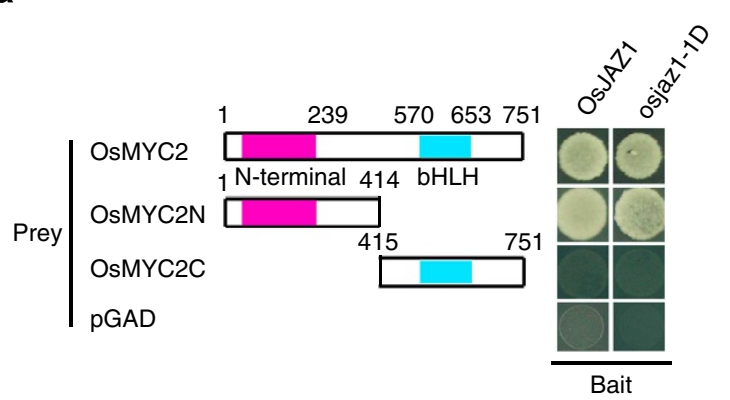

C

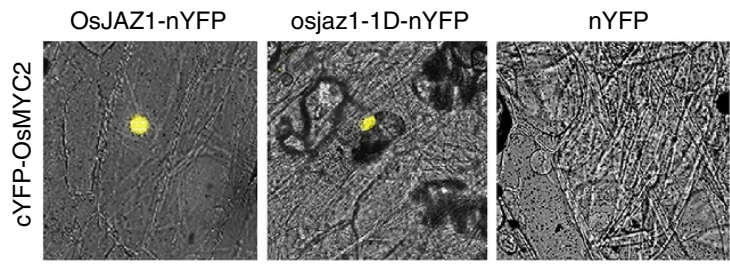

b

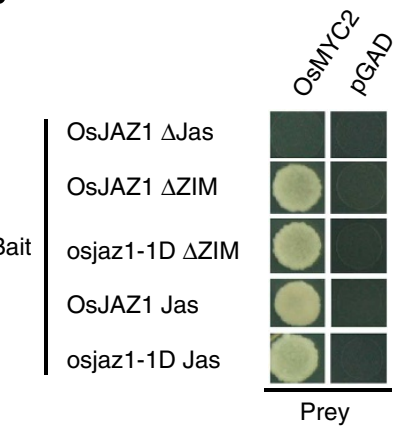

d

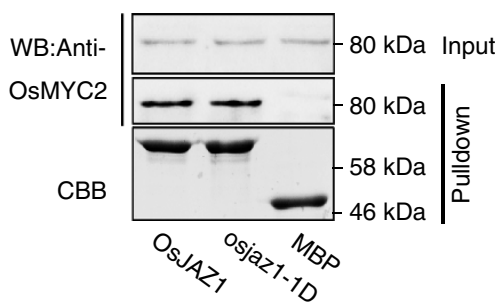

Figure 7 | Interaction of OsJAZ1/osjaz1-1D and OsMYC2 in vitro and in vivo. (a) $\mathrm{Y} 2 \mathrm{H}$ analysis showing the requirement of the $\mathrm{N}$-terminal conserved region of OsMYC2 for its interaction with OsJAZ1/osjaz1-1D. (b) Y2H analysis showing the requirement of the Jas domain in OsJAZ1/osjaz1-1D for its interaction with OsMYC2. (c) Bimolecular fluorescence complementation assays showing the interaction between OsMYC2 and OsJAZ1/osjaz1-1D. (d) In vitro pull-down assays showing the interaction between OsMYC2 and OsJAZ1/osjaz1-1D. The precipitated OsMYC2 was detected by anti-OsMYC2 antibody. Molecular weight of proteins, OsMYC2 $=81 \mathrm{kDa}$, OsJAZ1/osjaz1-1D $=65 \mathrm{kDa}, \mathrm{MBP}=51 \mathrm{kDa}$. WB, western blot, CBB, Coomassie brilliant blue staining.

vector to fuse with GUS. To generate the OsJAZ1 pro:osjaz1-1D gDNA transgenic lines, a $6.6-\mathrm{kb}$ genomic sequence containing the $3.0-\mathrm{kb}$ promoter and $3.6-\mathrm{kb}$ genomic sequence of the mutated $E G 2$ gene from the eg2-1D mutant was cloned into $p$ CAMBIA1301. To generate $35 S_{\text {pro: }}$ OsJAZ1-GUS and $35 S_{\text {pro:osjaz1-1D-GUS }}$ lines, OsJAZ1 and osjaz1-1D cDNAs were amplified from wild-type and eg2-1Dmutant background, respectively, and then cloned into pCAMBIA1301. To generate $35 S_{\text {pro }}: E G 1$ lines, the cDNA of EG1 was cloned into the $P H B$ vector (kindly provided by Prof. H.Q.Yang). To generate $E G 1_{\text {pro }}: D G L$ lines, the 2-kb EG1 promoter region and cDNA of DGL were cloned into pCAMBIA1301. See Supplementary Table 2 for all the primers used for making the constructs. All transgenic plants of rice and Arabidopsis were generated by Agrobacteriummediated transformation and confirmed by PCR genotyping and qRT-PCR (Supplementary Fig. 15a-h)

Map-based cloning of EG1 and EG2. To map EG1, crosses were made between eg1-3 and Guang-lu-ai 4 (wild type, O. sativa L. ssp. indica). Plants with abnormal spikelet phenotypes were selected from the $\mathrm{F}_{2}$ populations for mapping. For mapping of eg2-1D, crosses were made between eg2-1D and 9311 (wild type, O. sativa L. ssp. indica), and the wild type-like plants in the $\mathrm{F}_{2}$ populations were used for mapping. In-Del molecular markers (Primers are described in Supplementary Table 2) were designed based on the sequence difference between japonica and indica genomes.

Rice protoplast transformation. Rice protoplast transformation was performed by using polyethylene glycol (PEG)-mediated transfections as described by Bart et al. ${ }^{58}$. Stem tissues of seedlings (14 days after germination) were cut into $0.5 \mathrm{~mm}$ pieces and incubated in enzyme solution ( $0.6 \mathrm{M}$ mannitol, $10 \mathrm{mM}$ MES, pH 5.7, $1.5 \%$ cellulase RS, $0.75 \%$ macerozyme, $0.1 \% \mathrm{BSA}, 1 \mathrm{mM} \mathrm{CaCl}$ ) for $4 \mathrm{~h}$ in the dark under shaking (40 r.p.m.). After the removal of the enzyme solution, tissues were incubated in W5 solution ( $154 \mathrm{mM} \mathrm{NaCl}, 125 \mathrm{mM} \mathrm{CaCl}_{2}, 5 \mathrm{mM} \mathrm{KCl}, 2 \mathrm{mM} \mathrm{MES}$, $\mathrm{pH}$ 5.7) for $1 \mathrm{~h}$ under shaking ( $40 \mathrm{r}$.p.m.) to release protoplasts. After incubation, the incubated mixture was passed through a $35 \mu \mathrm{m}$ nylon mesh filter, mixed with one volume of W5 solution and then centrifuged for $5 \mathrm{~min}$ at 1500 r.p.m. to pellet the protoplasts. Cells were re-suspended in Mmg solution (0.6 M mannitol, $15 \mathrm{mM}$ $\mathrm{MgCl}_{2}, 4 \mathrm{mM}$ MES, pH 5.7) for PEG-mediated transformation at $10^{6}$ cells per ml. For transformation, $40 \%$ PEG (0.6 M mannitol, $100 \mathrm{mM} \mathrm{CaCl}_{2}, 40 \%$ v/v PEG 4000) and $10 \mu \mathrm{g}$ plasmid were added to the protoplasts for 15 minutes at room temperature. Cells were washed $1 \times$ with 10 volumes of W5 and then re-suspended in W5 solution at $28^{\circ} \mathrm{C}$ in the dark overnight.
Protein subcellular localization analysis. $35 S_{p r o}$ :EG1-GFP (full-length EG1 cDNA fused with GFP) was generated in PCAMBIA1301 (primers are described in Supplementary Table 2) and expressed in rice protoplasts using PEG-mediated transfections.

The localization of EG1-GFP was analysed in Nicotiana benthamiana (tobacco) leaves after agroinfiltration ${ }^{59}$. Briefly, overnight cultured Agrobacteria containing the plasmid were collected and re-suspended in Murashige and Skoog liquid medium that contained $10 \mu \mathrm{M}$ MES (pH 5.6) and $200 \mu \mathrm{M}$ Acetosyringone to $\mathrm{OD}_{600}=0.6$, and then incubated for $3 \mathrm{~h}$ at room temperature before being infiltrated into the young leaf of tobacco. Before images were taken, the plants were grown for about $48 \mathrm{~h}$ under weak light conditions.

Images were captured by a Confocal Laser Scanning Microscope equipped with an argon/krypton laser (Leica TCS SP5). GFP fluorescent and chlorophyll autofluorescent signals were imaged at the excitation wavelength of $488 \mathrm{~nm}$, and emission wavelength of $505-530 \mathrm{~nm}$ and $650-798 \mathrm{~nm}$, respectively.

Endogenous JA measurement and MeJA treatment. Endogenous JA was measured according to the method by Fu et al. ${ }^{60}$. Briefly, about $200 \mathrm{mg}$ fresh weight of plant tissue from the wild type and eg1-3 were homogenized, and endogenous JA was extracted for $24 \mathrm{~h}$ in methanol, with ${ }^{2} \mathrm{H}_{5}-\mathrm{JA}$ (CDN Isotopes) added as internal standard. JA levels were analysed by a LC/MS/MS system, which consisted of an Acquity Ultra Performance Liquid Chromatograph (Acquity UPLC; Waters) and a triple quadruple tandem mass spectrometer (Quattro Premier XE; Waters). Three biological replicates were performed for each group.

MeJA treatment of rice spikelet was performed 2 weeks before inflorescence initiation. MeJA (95\%; Sigma-Aldrich) was dissolved in 0.05\% aqueous Tween-20 to $50 \mu \mathrm{M}$, and then added into the soil medium in which eg1-3 plants had been growing. For seedling treatment, 3-day-old seedlings of 9522, eg1-3 and eg2-1D were transferred to $1 / 2$ Murashige and Skoog medium containing 1 or $10 \mu \mathrm{M}$ MeJA, and grew for 5 days before harvesting.

Gene expression analyses. For qRT-PCR analysis, total RNA was isolated with Plant Total RNA Mini Kit (TianGen), and first strand cDNA was synthesized from $1 \mu \mathrm{g}$ of total RNA with M-MLV reverse transcriptase (PrimeScript RT reagent Kit, Takara). GRT-PCR was performed with the CFX384 real-time PCR detection system (Bio-Rad) using the SYBR Green mix (Bio-Rad) (Primers are described in Supplementary Table 2).

For RNA in situ hybridizations, digoxygenin-labeled antisense and sense probes of EG1, OsMADS1, OsMADS7 and OsMADS8 were transcribed in vitro using the in vitro transcription kit according to the user manual (ACCU-CHEK). 
a

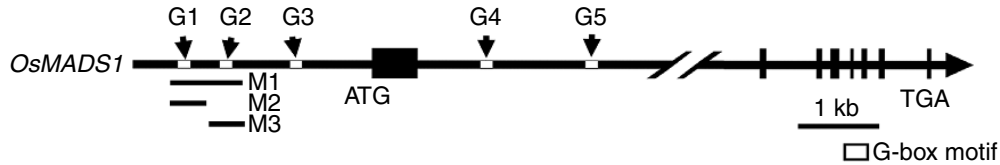

b

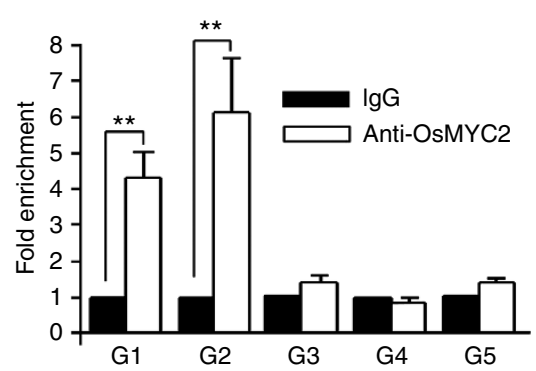

c

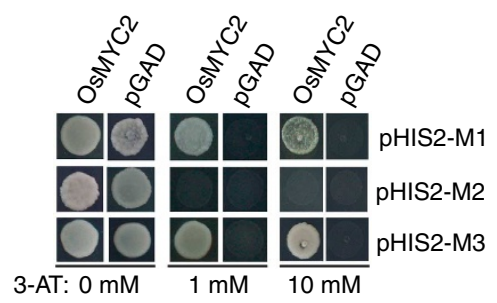

d

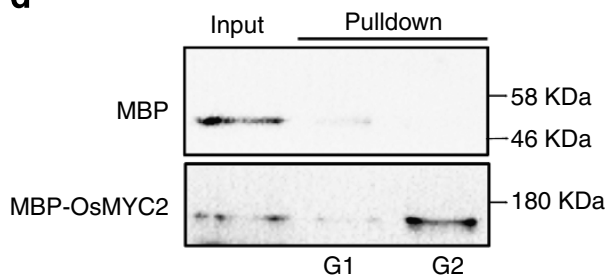

e

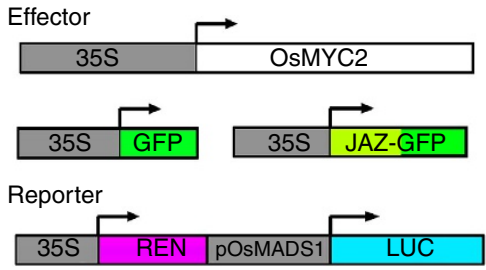

POSMADS1-LUC

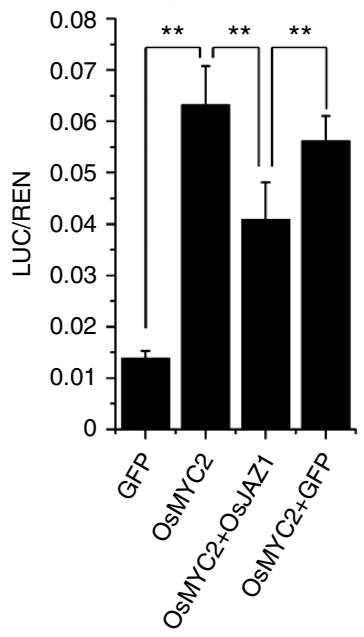

Figure 8 | OsMYC2 regulates the transcription of OsMADS1. (a) Diagram of the OsMADS1 genomic region showing the five G-box motifs, G1 to G5. Black boxes are exons. (b) qChIP-PCR results showing that the promoter fragments G1 and G2 can be amplified from IP pulled down by the anti-OsMYC2 antibody. In the control, immunoglobulin $\mathrm{G}$ was set as 1. Each data point represents the average of three biological repeats, and error bars indicate s.d. Asterisks indicate significant difference between samples (Student's $t$-test $P$-values, ${ }^{\star} P<0.05$; ${ }^{\star \star} P<0.01$ ). (c) Yeast one-hybrid analysis showing interaction between OsMYC2 and the M2 region of OsMADS1 promoter. M1, M2, M3 are shown in (a). 3-amino-1,2,4-triazole, 3-amino-1,2,4-triazole. (d) DNA-binding assays showing OsMYC2's binding to G2 in the promoter of OsMADS1. The precipitated OsMYC2 was detected by anti-OsMYC2 antibody. Molecular weight of proteins, MBP-OsMYC2 $=123 \mathrm{kDa}, \mathrm{MBP}=51 \mathrm{kDa}$. (e) Transient dual-LUC reporter gene assays showing OsJAZ1's ability to inhibit OsMYC2's transcription activity on the OsMADS1 promoter. Constructs used in the transient transactivation assays are shown in the upper panel. Five biological repeats were performed for each sample, error bars indicate s.d. Asterisks indicate significant difference between samples (Student's $t$-test $P$-values, $\left.{ }^{\star} P<0.05 ;{ }^{\star \star} P<0.01\right)$. There was no significant difference between OsMYC2 + GFP and OsMYC2 (Student's $t$-test $P$-values, $P>0.05$ ).

Floral primordia of young inflorescences were fixed using formalin/acetic acid/ alcohol fixative solution at $4{ }^{\circ} \mathrm{C}$ overnight, dehydrated and embedded in Paraplast Plus paraffin (Sigma). In situ hybridization procedures used were from $\mathrm{Li}$ et al. ${ }^{55}$. Sequences of the probes used, that is, EG1, OsMADS1, OsMADS7 and OsMADS8, were from previous reports ${ }^{36,39,55}$. Images were captured with a Leica DM750M fluorescence microscope.

Procedure for GUS staining of $E G 1_{\text {pro: }}$ GUS lines was modified from a previously published protocol ${ }^{61}$. Samples were incubated in GUS staining solution $(50 \mathrm{mM}$ $\mathrm{NaPO}_{4}$ buffer, $\mathrm{pH} 7.0,10 \mathrm{mg} \mathrm{ml}^{-1} \mathrm{X}$-Gluc and $0.02 \%$ (v/v) Triton X-100), at $37^{\circ} \mathrm{C}$ in the dark. After staining, samples were washed with $70 \%$ ethanol at room temperature. The GUS staining images were taken by a Leica MZ8 dissecting microscope.

Detection of protein degradation in vivo. Transgenic $35 S_{\text {pro }}$ OsJAZ1-GUS and $35 S_{\text {pro: }}$ osjaz1-1D-GUS plants were treated with MeJA $(100 \mu \mathrm{M})$ with or without the proteasome inhibitor MG132 $(100 \mu \mathrm{M})(\mathrm{MERCK})$ for $1 \mathrm{~h}$. Histochemical GUS staining of roots was performed as described previously ${ }^{61}$, and results were observed under a Leica MZ8 dissecting microscope. The quantity of the GUSfusing protein was also detected by western blot using anti- $\beta$ glucuronidase (GUS) antibody (Abcam, 1:2,000 dilution).
Yeast one/two-hybrid assays. For $\mathrm{Y} 2 \mathrm{H}$ assays, the full-length or specific domains of OsCOI1a, OsCOI1b, OsCOI1c, OsJAZ1, osjaz1-1D and OsMYC2 were amplified and cloned into the $\mathrm{Y} 2 \mathrm{H}$ vectors $\mathrm{p} G B K T 7$ and $\mathrm{p} G A D T 7$ (Clontech), respectively (primers are described in Supplementary Table 2). Y2H experiments were then carried out following the manual of Matchmaker Gold Yeast Two-Hybrid System (Clontech). Detection of protein-protein interactions between OsJAZ1 and OsCOI1a/OsCOI1b/OsCOI1c were performed in $\mathrm{SD} /$-Trp/-Leu/-His/-Ade medium containing $30 \mu \mathrm{M} \mathrm{JA}$, MeJA or Coronatine, all of which were dissolved in $1 \%$ methanol.

For yeast one-hybrid assays, specific fragments of the OsMADS1 promoter region were amplified and cloned into the yeast one-hybrid $(\mathrm{Y} 1 \mathrm{H})$ vector $\mathrm{pHIS} 2$ (Clontech) (Primers are described in Supplementary Table 2). Y1H experiments were then carried out following the transformation protocol in the Yeastmaker Yeast Transformation System 2 user manual (Clontech). Detection of protein-DNA interactions between OsMYC2 and OsMADS1 promoter region was performed in $\mathrm{SD} /$-Trp/-Leu/-His medium containing 0,1 or $10 \mathrm{mM}$ of 3-amino-1,2,4-triazole.

Bimolecular fluorescence complementation assays. The cDNAs of $O s M Y C 2$, $O s J A Z 1$, osjaz1-1D and OsMYC2 were individually cloned into pSAT1-nEYFP-N1 


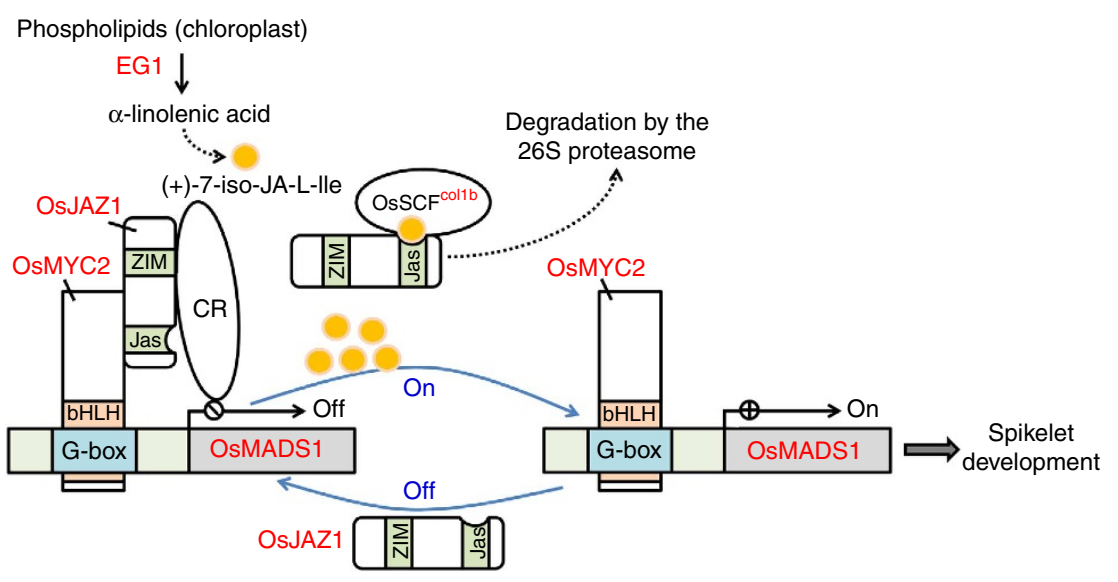

Figure 9 | A working model of the role of JA in regulating rice spikelet development. EG1-mediated JA biosynthesis is required for rice spikelet development. Upon perception of the JA signal, OsCOI1b recruits OsJAZ1 to the SCFCOI1 complex for ubiquitination and degradation through the $26 \mathrm{~S}$ proteasome. As a result, OsMYC2 is released from the OsJAZ1-OsMYC2 complex and proceeds with its function in activating the expression of downstream genes, such as the E-class gene OsMADS1. Proteins investigated in this study are in red. bHLH, basic-helix-loop-helix domain;

CR: co-repressors.

and $\mathrm{p} S A T 1-c E Y F P-C 1-B$ vectors that contained either amino- or carboxy-terminal EYFP fragments (Primers are described in Supplementary Table 2). The method for bimolecular fluorescence complementation assays using onion epidermal cells was modified from Lee et al. ${ }^{62}$. After removal of the dry outer layer, whole onion was sterilized by $2 \% \mathrm{NaOCl}$ and washed with sterilized $\mathrm{H}_{2} \mathrm{O}$ for five times. The onion was cut into $2 \times 2 \mathrm{~cm}$ squares, and placed in a plate with Murashige and Skoog medium containing $40 \mathrm{gl}^{-1}$ D-Mannitol for $4 \mathrm{~h}$. For transformation, $5 \mu \mathrm{g}$ of each plasmid DNA was coated with 1.0- $\mu \mathrm{m}$ gold particles (Bio-Rad) and delivered into onion epidermal cells using a PDS-100/He Particle Delivery System (Bio-Rad). Images were captured one night after transformation by a Confocal Laser Scanning Microscope equipped with an argon/krypton laser (Leica TCS SP5). YFP fluorescence was imaged at the excitation wavelength of $514 \mathrm{~nm}$ and emission wavelength of $525-546 \mathrm{~nm}$.

OsMYC2 polyclonal antibody preparation. A 120 -amino acid OsMYC2-specific peptide, which consisted of amino acids 16-84, 320-345 and 396-420 of the protein, was synthesized in vitro using optimized codons for E. coli genes (the sequence is described in Supplementary Table 3) and cloned into the $P M A L$ $c 2 X$ vector (NEB) to fuse with MBP. The recombinant protein was expressed in $E$. coli DE3 (BL21) (Novagen) and purified using Affinity Resin (Clontech) to produce rabbit polyclonal antibodies (prepared by Abclonal of China). The antibody (1:1,000 dilution) was tested by immunoblot analysis using total proteins and nuclear proteins extracted from wild-type spikelet at late stage Sp8, which detected a single band of the expected size at $81 \mathrm{kD}$ (Supplementary Fig. 15i).

In vitro pull-down assays. For in vitro pull-down experiment, full-length cDNAs of $O s J A Z 1$ and osjaz1-1D were synthesized in vitro using optimized codons for E. coli genes (primers are described in Supplementary Table 2 and sequences are described in Supplementary Table 3 ) and cloned into the $\mathrm{p} M A L-c 2 X$ vector (NEB) to fuse with maltose binding protein (MBP). A quantity of $0.5 \mathrm{mg}$ total proteins extracted from wild-type rice spikelet at stage Sp8 was incubated with $1 \mu \mathrm{g}$ immobilized MBP, MBP-OsJAZ1 or MBP-osjaz1-1D at $4^{\circ} \mathrm{C}$ for $1 \mathrm{~h}$. Proteins retained on the beads were eluted and separated by SDS-PAGE, and stained with Coomassie Brilliant R250 (Fisher Scientific) or subjected to immunoblotting with the anti-OsMYC2 antibody (1:1,000 dilution) (Uncropped images of blots are shown in Supplementary Fig. 16).

ChIP assays. Chromatin immunoprecipitation (ChIP) assays involved the procedures described by Li. et al. ${ }^{55}$. Chromatin was isolated from rice spikelets at stage Sp4-Sp8 and sonicated, and the solubulized chromatin was immunoprecipitated by either anti-OsMYC2 antibody or rabbit immunoglobulin $\mathrm{G}$ at $4^{\circ} \mathrm{C}$ overnight. After immunoprecipitation, DNA was collected by protein A/G Plus Agarose (Calbiochem). For each qRT-PCR reaction, the recovered DNA from immunoprecipitation was used as template. Three biological replicates were included. All primers used in the ChIP assays are listed in Supplementary Table 2.

DNA-binding assays. Protein-DNA-binding assays were performed as described by Bai et al. ${ }^{63}$. Full-length OsMYC2 cDNA was synthesized in vitro using optimized codons for E.coli genes (Sequences are described in Supplementary Table 3) and cloned into the $\mathrm{pMAL}-c 2 X$ vector to fuse with MBP. MBP and MBP-OsMYC2 were expressed and affinity-purified from E. coli. (BL21) using amylose resin (NEB). The
DNA probe fragments were amplified from genomic DNA of wild type by PCR using biotin-labeled primers (primers are described in Supplementary Table 2). Proteins were pulled down using streptavidin-agarose beads (Sigma) that were bound to the DNA probe fragments, and detected by western blot with anti-MBP antibody (EarthOx, 1:2,000 dilution) (uncropped images of blots are shown in Supplementary Fig. 16).

Protoplast transient reporter gene assays. To generate the effector and reporter constructs, $p$ GreenII- 0000 vector containing the $35 S$ promoter (kindly provided by $\mathrm{Hao} \mathrm{Yu}$ ) and $p$ GreenII-0800-LUC (kindly provided by Hongquan Yang) were used $^{64}$. All primers used for generating constructs for transient transactivation assays are listed in Supplementary Table 2. The transient assays were performed in rice protoplasts using PEG-mediated transfections (See the section of Rice Protoplast Transformation), and LUC and REN activities were analysed using the Dual-Luciferase reporter kit according to the user manual (Promega). Absolute LUC/REN was measured in a Turner 20/20 luminometer (Promega).

\section{References}

1. Group, G. P. W. Phylogeny and subfamilial classification of the grasses (Poaceae). Ann. Missouri. Bot. Gard. 88, 373-457 (2001).

2. Schmidt, R. J. \& Ambrose, B. A. The blooming of grass flower development. Curr. Opin. Plant Biol. 1, 60-67 (1998).

3. Tanaka, W., Pautler, M., Jackson, D. \& Hirano, H. Y. Grass meristems II: inflorescence architecture, flower development and meristem fate. Plant. Cell Physiol. 54, 313-324 (2013).

4. Yuan, Z. et al. RETARDED PALEA1 controls palea development and floral zygomorphy in rice. Plant Physiol. 149, 235-244 (2009).

5. Yamaguchi, N. et al. A molecular framework for auxin-mediated initiation of flower primordia. Dev. Cell 24, 271-282 (2013).

6. Liu, C., Thong, Z. \& Yu, H. Coming into bloom: the specification of floral meristems. Development 136, 3379-3391 (2009).

7. Wagner, D. Flower morphogenesis: timing is key. Dev. Cell 16, 621-622 (2009).

8. Ferrandiz, C., Gu, Q., Martienssen, R. \& Yanofsky, M. F. Redundant regulation of meristem identity and plant architecture by FRUITFULL, APETALA1 and CAULIFLOWER. Development 127, 725-734 (2000).

9. Causier, B., Schwarz-Sommer, Z. \& Davies, B. Floral organ identity: 20 years of ABCs. Semin. Cell Dev. Biol. 21, 73-79 (2010).

10. Ikeda-Kawakatsu, K., Maekawa, M., Izawa, T., Itoh, J. \& Nagato, Y. ABERRANT PANICLE ORGANIZATION 2/RFL, the rice ortholog of Arabidopsis LEAFY, suppresses the transition from inflorescence meristem to floral meristem through interaction with APO1. Plant J. 69, 168-180 (2012).

11. Kobayashi, K. et al. Inflorescence meristem identity in rice is specified by overlapping functions of three AP1/FUL-like MADS box genes and PAP2, a SEPALLATA MADS box gene. Plant Cell 24, 1848-1859 (2012).

12. Rao, N. N., Prasad, K., Kumar, P. R. \& Vijayraghavan, U. Distinct regulatory role for RFL, the rice $L F Y$ homolog, in determining flowering time and plant architecture. Proc. Natl Acad. Sci. USA 105, 3646-3651 (2008).

13. Jeon, J. S. et al. leafy hull sterile1 is a homeotic mutation in a rice MADS box gene affecting rice flower development. Plant Cell 12, 871-884 (2000). 
14. Malcomber, S. T. \& Kellogg, E. A. Heterogeneous expression patterns and separate roles of the SEPALLATA gene LEAFY HULL STERILE1 in grasses. Plant Cell 16, 1692-1706 (2004).

15. Chen, Z. X. et al. Morphogenesis and molecular basis on naked seed rice, a novel homeotic mutation of OsMADS1 regulating transcript level of AP3 homologue in rice. Planta 223, 882-890 (2006).

16. Chuck, G., Meeley, R. B. \& Hake, S. The control of maize spikelet meristem fate by the APETALA2-like gene indeterminate spikelet1. Genes Dev. 12, 1145-1154 (1998).

17. Chuck, G., Meeley, R. \& Hake, S. Floral meristem initiation and meristem cell fate are regulated by the maize AP2 genes ids1 and sid1. Development 135, 3013-3019 (2008)

18. Lee, D. Y., Lee, J., Moon, S., Park, S. Y. \& An, G. The rice heterochronic gene SUPERNUMERARY BRACT regulates the transition from spikelet meristem to floral meristem. Plant J 49, 64-78 (2007).

19. Lee, D. Y. \& An, G. Two AP2 family genes, Supernumerary Bract (SNB) and Osindeterminate spikelet 1 (OsIDS1), synergistically control inflorescence architecture and floral meristem establishment in rice. Plant J. 69, 445-461 (2012).

20. Acosta, I. F. \& Farmer, E. E. Jasmonates. Arabidopsis Book 8, e0129 (2010).

21. Browse, J. The power of mutants for investigating jasmonate biosynthesis and signalling. Phytochemistry 70, 1539-1546 (2009).

22. Browse, J. \& Howe, G. A. New weapons and a rapid response against insect attack. Plant Physiol. 146, 832-838 (2008)

23. Kessler, A., Halitschke, R. \& Baldwin, I. T. Silencing the jasmonate cascade: induced plant defenses and insect populations. Science 305, 665-668 (2004).

24. Wasternack, C. \& Hause, B. Jasmonates: biosynthesis, perception, signal transduction and action in plant stress response, growth and development. An update to the 2007 review in Annals of Botany. Ann. Bot. 111, 1021-1058 (2013).

25. Hyun, Y. et al. Cooperation and functional diversification of two closely related galactolipase genes for jasmonate biosynthesis. Dev. Cell 14, 183-192 (2008)

26. Ishiguro, S., Kawai-Oda, A., Ueda, J., Nishida, I. \& Okada, K. The DEFECTIVE IN ANTHER DEHISCIENCE gene encodes a novel phospholipase A1 catalyzing the initial step of jasmonic acid biosynthesis, which synchronizes pollen maturation, anther dehiscence, and flower opening in Arabidopsis. Plant Cell 13, 2191-2209 (2001)

27. Ellinger, D. et al. DONGLE and DEFECTIVE IN ANTHER DEHISCENCE1 lipases are not essential for wound- and pathogen-induced jasmonate biosynthesis: redundant lipases contribute to jasmonate formation. Plant Physiol. 153, 114-127 (2010).

28. Chini, A. et al. The JAZ family of repressors is the missing link in jasmonate signalling. Nature 448, 666-671 (2007).

29. Katsir, L., Schilmiller, A. L., Staswick, P. E., He, S. Y. \& Howe, G. A. COI1 is a critical component of a receptor for jasmonate and the bacterial virulence factor coronatine. Proc. Natl Acad. Sci. USA 105, 7100-7105 (2008).

30. Melotto, M. et al. A critical role of two positively charged amino acids in the Jas motif of Arabidopsis JAZ proteins in mediating coronatine- and jasmonoyl isoleucine-dependent interactions with the COI1 F-box protein. Plant J. 55, 979-988 (2008)

31. Sheard, L. B. et al. Jasmonate perception by inositol- phosphate- potentiated COI1-JAZ co-receptor. Nature 468, 400-405 (2010).

32. Thines, B. et al. JAZ repressor proteins are targets of the $\mathrm{SCF}^{\mathrm{COI} 1}$ complex during jasmonate signalling. Nature 448, 661-665 (2007).

33. Xie, D. X., Feys, B. F., James, S., Nieto-Rostro, M. \& Turner, J. G. COI1: an Arabidopsis gene required for jasmonate-regulated defense and fertility. Science 280, 1091-1094 (1998).

34. Xu, L. et al. The SCF ${ }^{\mathrm{COI} 1}$ ubiquitin-ligase complexes are required for jasmonate response in Arabidopsis. Plant Cell 14, 1919-1935 (2002).

35. Yan, J. et al. The Arabidopsis CORONATINE INSENSITIVE1 protein is a jasmonate receptor. Plant Cell 21, 2220-2236 (2009).

36. Li, H. et al. A putative lipase gene EXTRA GLUME1 regulates both empty-glume fate and spikelet development in rice. Plant J. 57, 593-605 (2009).

37. Lee, H. Y. et al. Oryza sativa COI homologues restore jasmonate signal transduction in Arabidopsis coil-1 mutants. PLoS One 8, e52802 (2013).

38. Cui, R. et al. Functional conservation and diversification of class E floral homeotic genes in rice (Oryza sativa). Plant J. 61, 767-781 (2010).

39. Gao, X. et al. The SEPALLATA-like gene OsMADS34 is required for rice inflorescence and spikelet development. Plant Physiol. 153, 728-740 (2010).

40. Kobayashi, K., Maekawa, M., Miyao, A., Hirochika, H. \& Kyozuka, J. PANICLE PHYTOMER2 (PAP2), encoding a SEPALLATA subfamily MADS-box protein, positively controls spikelet meristem identity in rice. Plant Cell Physiol. 51, 47-57 (2010).

41. Lorenzo, O., Chico, J. M., Sanchez-Serrano, J. J. \& Solano, R. JASMONATEINSENSITIVE1 encodes a MYC transcription factor essential to discriminate between different jasmonate-regulated defense responses in Arabidopsis. Plant Cell 16, 1938-1950 (2004).
42. Kazan, K. \& Manners, J. M. MYC2: The Master in Action. Mol. Plant 6, 686-703 (2013).

43. Fernandez-Calvo, P. et al. The Arabidopsis bHLH transcription factors MYC3 and MYC4 are targets of JAZ repressors and act additively with MYC2 in the activation of jasmonate responses. Plant Cell 23, $701-715$ (2011)

44. Feys, B., Benedetti, C. E., Penfold, C. N. \& Turner, J. G. Arabidopsis mutants selected for resistance to the phytotoxin Coronatine are male sterile, insensitive to methyl Jasmonate, and resistant to a bacterial pathogen. Plant Cell 6, 751-759 (1994).

45. Li, L. et al. The tomato homolog of CORONATINE-INSENSITIVE1 is required for the maternal control of seed maturation, jasmonate-signaled defense responses, and glandular trichome development. Plant Cell 16, 126-143 (2004).

46. Acosta, I. F. et al. Tasselseed1 is a lipoxygenase affecting jasmonic acid signalling in sex determination of maize. Science 323, 262-265 (2009).

47. Browse, J. Jasmonate: preventing the maize tassel from getting in touch with his feminine side. Sci. Signal. 2, pe9 (2009).

48. Yan, Y. et al. Disruption of OPR7 and OPR8 reveals the versatile functions of jasmonic acid in maize development and defense. Plant Cell 24, 1420-1436 (2012).

49. Zhang, D. B. \& Yuan, Z. Molecular control of grass inflorescence development. Annu. Rev. Plant. Biol. (doi:10.1146/annurev-arplant-050213-040104).

50. Yoshida, A., Suzaki, T., Tanaka, W. \& Hirano, H. Y. The homeotic gene long sterile lemma (G1) specifies sterile lemma identity in the rice spikelet. Proc. Natl Acad. Sci. USA 106, 20103-20108 (2009).

51. Tanaka, W. et al. The YABBY gene TONGARI-BOUSHI1 is involved in lateral organ development and maintenance of meristem organization in the rice spikelet. Plant Cell 24, 80-95 (2012).

52. Zhang, D. B., Yuan, Z., An, G., Dreni, L., Hu, J. P. \& Kater, M. Panicle Development. Genetics and Genomics of Rice, Plant Genetics and Genomics: Crops and Models 5, 279-295 (2013).

53. Li, H. et al. The AGL6-like gene OsMADS6 regulates floral organ and meristem identities in rice. Cell. Res. 20, 299-313 (2010).

54. Ohmori, S. et al. MOSAIC FLORAL ORGANS1, an AGL6-like MADS box gene, regulates floral organ identity and meristem fate in rice. Plant Cell 21, 3008-3025 (2009).

55. Li, H. et al. Rice MADS6 interacts with the floral homeotic genes SUPERWOMAN1, MADS3, MADS58, MADS13, and DROOPING LEAF in specifying floral organ identities and meristem fate. Plant Cell 23, 2536-2552 (2011).

56. Thompson, B. E. et al. bearded-ear encodes a MADS box transcription factor critical for maize floral development. Plant Cell 21, 2578-2590 (2009).

57. Ikeda, K., Sunohara, H. \& Nagato, Y. Developmental course of inflorescence and spikelet in rice. Breed. Sci. 54, 147-156 (2004).

58. Bart, R., Chern, M., Park, C. J., Bartley, L. \& Ronald, P. C. A novel system for gene silencing using siRNAs in rice leaf and stem-derived protoplasts. Plant Methods 2, 13 (2006).

59. Voinnet, O., Rivas, S., Mestre, P. \& Baulcombe, D. An enhanced transient expression system in plants based on suppression of gene silencing by the p19 protein of tomato bushy stunt virus. Plant J. 33, 949-956 (2003).

60. Fu, J., Chu, J., Sun, X., Wang, J. \& Yan, C. Simple, rapid, and simultaneous assay of multiple carboxyl containing phytohormones in wounded tomatoes by UPLC-MS/MS using single SPE purification and isotope dilution. Anal. Sci. 28, 1081-1087 (2012).

61. Jefferson, R. A., Kavanagh, T. A. \& Bevan, M. W. GUS fusions: betaglucuronidase as a sensitive and versatile gene fusion marker in higher plants. EMBO J. 6, 3901-3907 (1987).

62. Lee, L. Y., Fang, M. J., Kuang, L. Y. \& Gelvin, S. B. Vectors for multi-color bimolecular fluorescence complementation to investigate protein-protein interactions in living plant cells. Plant Methods 4, 24 (2008).

63. Bai, M. Y. et al. Brassinosteroid, gibberellin and phytochrome impinge on a common transcription module in Arabidopsis. Nat. Cell. Biol. 14, 810-817 (2012).

64. Hellens, R. P. et al. Transient expression vectors for functional genomics, quantification of promoter activity and RNA silencing in plants. Plant Methods 1, 13 (2005).

\section{Acknowledgements}

We thank Prof. Y.B. Xue (Institute of Genetics and Developmental Biology, Chinese Academy of Sciences) for providing seeds of the eg1-1 mutant, J.H. Tong (Hu Nan Agriculture University) for measurement of endogenous JA content, Hao Yu (National University of Singapore) for providing pGreenII-0000 vectors, and HQ Yang (Shanghai Jiao Tong University) for providing $P H B$ and pGreenII-0800 vectors and Arabidopsis Biological Resource Center for providing the T-DNA insertion line (SALK_025432) of $D A D 1$. This work was supported by funds from National Natural Science Foundation of China (31230051, 30971739, 31270222 and 31110103915); National Key Basic Research 
Developments Program, Ministry of Science and Technology, China (2013CB126902 and 2011CB100101); China Innovative Research Team, Ministry of Education; 111 Project (B14016); the 863 High-Tech Project, Ministry of Science and Technology, China (2011AA10A101 and 2012AA10A302).

\section{Author contributions}

Z.Y., M.C. and Z.L. conducted the mutant screen, identified eg1-3 and eg2-1D and performed preliminary genetic and phenotypes analyses. X.Z. performed genetic analyses of the eg1-3, eg2-1D and eg1-1 osmads1-1mutants. Q.C. mapped the EG1 and EG2 genes and conducted follow-up studies on the function of the EG1 and EG2 proteins. C.Y. performed in situ hybridization analysis. Z.Y., W.L. and D.Z. conceived the study, supervised the work and analysed the data. J.H. and X.Z. participated in the project discussions. D.Z., J.H., Q.C. and Z.Y. co-wrote the manuscript.

\section{Additional information}

Accession codes: The genomic sequence of EG1, EG2 (OsJAZ1) and OsMYC2 have been deposited in GRAMENE (http://www.gramene.org/) as LOC_Os01g67430, LOC_Os04g55920, and LOC_Os10g42430, respectively.

Supplementary Information accompanies this paper at http://www.nature.com/ naturecommunications

Competing financial interests: The authors declare no competing financial interests.

Reprints and permission information is available online at http://www.npg.nature.com/ reprintsandpermissions/

How to cite this article: Cai, Q. et al. Jasmonic acid regulates spikelet development in rice. Nat. Commun. 5:3476 doi: 10.1038/ncomms4476 (2014). 